\title{
Estilos de vida, hábitos dietéticos y prevalencia del sobrepeso y la obesidad en una población infantil
}

\author{
Á. Edo Martínez ${ }^{\mathrm{a}}$, I. Montaner Gomis ${ }^{\mathrm{b}}$, A. Bosch Moraga ${ }^{\mathrm{a}}$, \\ MR. Casademont Ferrer ${ }^{\mathrm{a}}$, MT. Fábrega Bautista ${ }^{\mathrm{a}}, \mathrm{A}^{\mathrm{A}}$. Fernández Buenoc, \\ M. Gamero Garcíac, MA. Ollero Torres ${ }^{c}$ \\ aPediatra. CAP Carmel (Institut Català de la Salut). Barcelona. España. \\ ${ }^{b}$ Médico de Familia. CAP Carmel (Institut Català de la Salut). Barcelona. España. \\ 'DUE. CAP Carmel (Institut Català de la Salut). Barcelona. España.
}

\section{Resumen}

Objetivos: conocer la prevalencia del sobrepeso y la obesidad en la población infantil, las características de sus hábitos dietéticos, su estilo de vida y otros determinantes asociados.

Material y métodos: diseño: estudio descriptivo transversal. Emplazamiento: Centro de Atención Primaria, ámbito urbano. Participantes: niños $>6$ y $<10$ años del ABS El Carmel (Barcelona) $(n=716)$.

Criterios de selección: muestra aleatoria $-n=340$; prevalencia: $15 \%$; intervalo de confianza (IC): 95\%; precisión: 3\%- de la base de datos SIAP (Sistema de Información en Atención Primaria). No se establecieron criterios de exclusión. Sin respuesta: $n=63$ (95\%: errores de filiación; 5\%: falta de interés).

Mediciones principales: peso y talla -para el índice de masa corporal (IMC)-, test rápido Krece Plus y test corto de actividad física Krece Plus (para hábitos dietéticos y estilo de vida). Análisis descriptivo y bivariado: chi al cuadrado, test de la t de Student, test de Anova, test de correlación y regresión logística.

Resultados: 276 encuestas (81,4\%). Prevalencia del sobrepeso: 11,59\% (IC 95\%: 7,81\%-15,32\%); prevalencia de la obesidad: 10,14\% (IC 95\%: 6,58\%-13,7\%). Hábitos alimentarios: 52,3\% tiene una alimentación óptima y 4,4\% presenta hábitos inadecuados. Estilo de vida: malo: 58,7\%; bueno: 3,6\%. La media del IMC es superior $(p<0,05)$ en los niños más inactivos, cuando el nivel de estudios de la madre es bajo o si nacieron fuera de España. El sobrepeso y la obesidad infantiles son 3,6 veces superiores (IC 95\%: 1,37-8,05) si el padre es obeso y 3,1 veces superiores (IC 95\%:1,19-7,31) si lo es la madre.

Conclusiones: el estudio proporciona una línea de base para establecer las directrices y actividades del programa de intervención comunitaria para la prevención de la obesidad infantil.

Palabras clave: Obesidad infantil. Hábitos dietéticos. Ejercicio físico. Atención Primaria.

Ángel Edo Martínez, aedo.bcn.ics@gencat.cat

Los autores declaran no presentar conflictos de intereses en relación con la preparación y publicación de este artículo. 


\section{Lifestyle, dietetic habits and overweight and obesity prevalence in a pediatric population}

\section{Abstract}

Objectives: to determine the prevalence of overweight and obesity in children, characteristics of their dietary habits, lifestyle and other associated determinants.

Material and method: design: cross-sectional study. Location: Primary Health Center, urban area. Participants: children $>6$ and $<10$ years of ABS El Carmel (Barcelona) $(n=716)$.

Selection criteria: random sample ( $n=340$; prevalence: 15\%; Cl: 95\%; accuracy: 3\%) from the database SIAP (System for Information in Primary Care), no exclusion criteria were established. No answer: $n=63$ (95\%: address error; 5\%: lack of interest).

Measurements: weight, height (for BMI), Quick Test and Short Test from Krece Plus (for diet and lifestyle). Descriptive and bivariate analysis: Chi-square, $t$-test, ANOVA test, correlation test and logistic regression.

Results: two hundred and seventy-six surveys (81.4\%). Overweight prevalence: $11.59 \%$ (95\% Cl: 7.81\%-15.32\%); obesity prevalence: 10.14\% (95\% Cl: 6.58\%-13.7\%). Food habits: $52.3 \%$ optimal feeding habits and $4.4 \%$ inadequate. Lifestyle: $58.7 \%$ bad lifestyle and $3.6 \%$ good lifestyle. The BMI mean was higher $(p<0.05)$ in inactive children, in low education level of mothers, or in born outside Spain. Overweight and obesity in children is 3.6 times higher $(95 \% \mathrm{Cl}$ : 1.37-8.05) if the father is obese and 3.1 times higher (95\% Cl:1.19-7.31) if the mother is obese.

Conclusions: the study provides a baseline for establishing the guidelines and activities of the community intervention program for preventing childhood obesity.

Key words: Child obesity. Dietary habits. Physical exercise. Primary care.

\section{Introducción}

En el Área Básica de Salud (ABS El Carmel) se inició hace varios años un proceso de orientación comunitaria con la metodología de la Atención Primaria Orientada a la Comunidad (APOC)'. La primera etapa de este ciclo de trabajo, conocida como examen preliminar, condujo a un listado con los principales problemas y necesidades de salud del barrio. Después de un proceso de discusión y consenso entre los miembros del equipo comunitario, se priorizó la obesidad infantil como uno de los problemas que más preocupaban al equipo de Atención Primaria (EAP) y a la población. Efectivamente, la obesidad es un problema emergente, especialmente entre los más jóvenes, y con un elevado potencial de morbimortalidad ${ }^{2,3}$, ya que se asocia a múltiples patologías, no sólo en la edad pediátrica, sino también en la vida adulta ${ }^{4,5,6}$. La tendencia al aumento en la prevalencia del sobrepeso y la obesidad en este grupo de edad es un hecho que preocupa no sólo a la población, sino también a la comunidad científica, lo que está movilizando esfuerzos por parte de las administraciones?. Otras consideraciones que se tuvieron en cuenta al priorizar fueron: la necesidad de un 
enfoque comunitario y multidisciplinar -ya que se involucran el ámbito familiar, comunitario, escolar, empresarial y sanitario- y ofrecer a la población infantil mayores posibilidades de prevención.

Una vez priorizado este problema de salud, y antes de planificar cualquier intervención, el EAP decidió realizar un estudio epidemiológico que permitiera conocer con más profundidad el problema y disponer de una línea de base para observar su evolución en el tiempo y evaluar los resultados de la futura intervención. Este estudio -previo a la implementación del programa de intervención- es lo que se denomina en la metodología APOC diagnóstico comunitario selectivo.

Los objetivos de este diagnóstico se definieron como: a) conocer la prevalencia del sobrepeso y la obesidad en la población infantil adscrita a la ABS El Carmel; b) averiguar las características y la calidad de sus hábitos alimentarios; y c) saber sus hábitos en la práctica de ejercicio físico. Como objetivo secundario se pretendía, además, conocer otros determinantes asociados al desarrollo de sobrepeso-obesidad en esta población.

\section{Material y métodos}

Se diseñó un estudio descriptivo transversal que se llevó a cabo de enero a junio de 2005. La posibilidad de realizar este es- tudio a toda la población infantil representaba una inversión de tiempo y recursos que no estaban disponibles, por lo que se decidió centrar el diagnóstico en un grupo de edad concreto, de fácil acceso para realizar las entrevistas y que se utilizaría en el futuro como indicativo de la evolución del problema. Por dicho motivo se eligió como población diana a los niños adscritos a la $A B S$ de $\geqslant 6 y<10$ años. No se establecieron criterios de exclusión. El número de niños para este grupo de edad era de 716 en enero de 2005. Se calculó el tamaño para una muestra representativa con los siguientes parámetros basados en la revisión bibliográfica ${ }^{8}$ : prevalencia esperada de la obesidad: $15 \%$; intervalo de confianza (IC): 95\%; y precisión: 3\%. Tamaño de la muestra (con corrección de poblaciones finitas y un $10 \%$ de no respuestas): 340 . La muestra se extrajo aleatoriamente de la base de datos SIAP (Sistema de Información en Atención Primaria).

Como variables principales del estudio se eligieron:

- Índice de masa corporal (IMC): se utilizaron las tablas de consenso AEP-SENC-SEEDO. Los individuos (niños y padres) fueron clasificados según su IMC en normal/sobrepeso/obesidad. Para los niños se consideró: normopeso < percentil 85; sobrepeso $>$ percentil 85 ; obesidad 
$>$ percentil 95. Para los adultos se consideró: sobrepeso IMC > 25 y obesidad IMC > 30 .

- Hábitos alimentarios: se midieron con el cuestionario rápido del estudio enKid (test rápido Krece Plus) ${ }^{10}$. Este test mide la adecuación de la alimentación a la dieta mediterránea (considerada correcta nutricionalmente). Según la puntuación del test, la alimentación del niño queda clasificada como de baja calidad, regular (necesita ajustes) y óptima.

- Actividad física: medida con el cuestionario rápido del estudio enKid (test corto de actividad física Krece Plus). Este test clasifica el estilo de vida basándose en la media diaria de horas que ven la televisión o juegan con videojuegos y las horas de deporte extraescolar por semana. Según la puntuación del test, el estilo de vida del niño se clasifica como malo, regular y bueno.

Como variables secundarias se eligieron: peso al nacer, lactancia materna (y tiempo), coexistencia de enfermedad crónica en el niño, presencia de obesidad o sobrepeso en los padres (a través del peso y la talla declarados y el cálculo del IMC), situación familiar (padres convivientes o no), escuela del niño, origen del niño y de los progenitores (y tiempo de residencia si era extranjero), nivel de instrucción del padre y de la madre, situación laboral del cabeza de familia y tipo de trabajo de éste.

Para la obtención de los datos se planificó la realización de una encuesta sobre la alimentación y el estilo de vida, así como un examen físico a la muestra de la población diana. A partir del listado de la muestra, el equipo de Pediatría organizó la forma de contactar con los padres. El resto de los miembros del equipo participó con diferente grado de compromiso según su disponibilidad. Durante los meses de enero a junio de 2005 se llevaron a cabo las encuestas y las mediciones somatométricas del estudio.

Los datos se introdujeron en formularios de formato Access ${ }^{\circledast}$ para su posterior tratamiento estadístico, realizado con el paquete SPSS ${ }^{\circledast}$. Se llevó a cabo el análisis de cada una de las variables estudiadas calculando las frecuencias, los porcentajes para las variables categóricas, la media, la desviación estándar y el IC del 95\% para las variables cuantitativas.

El análisis estadístico bivariado se efectuó comparando la variable principal del estudio (IMC de los niños) con el resto de las variables estudiadas. Este análisis se realizó desde dos perspectivas: por un lado, se utilizó el IMC como variable categórica -normopeso $(\mathrm{N})$, sobrepeso $(\mathrm{S})$ 
y obesidad (O)-; y, por otro, como variable cuantitativa continua (valor del IMC). Los test estadísticos utilizados fueron: chi al cuadrado para comparar porcentajes, test de la t y de Anova para la comparación de medias y test de correlación para comparar dos variables cuantitativas o sus equivalentes no paramétricos si no se cumplían las condiciones de aplicación. Se realizó una regresión logística tomando como variable dependiente la existencia o no de obesidad y, como variables independientes, aquellas que habían resultado con asociación estadísticamente significativa en el análisis bivariado. El nivel de significación se fijó en una $p \leqslant \leq$ 0,05 . En los casos en que los efectivos eran insuficientes para su tratamiento es- tadístico se agruparon en categorías las variables de: IMC (normopeso y sobrepeso + obesidad), horas de televisión (en menos y más de 3 horas) y horas de deporte (en ninguna, 1-3 horas y más de 3 horas de deporte a la semana).

\section{Resultados}

De una muestra inicial de 339 individuos, se realizaron 276 encuestas, lo que representa una participación del $81,4 \%$. Las no respuestas (63) corresponden en su mayor parte $(95 \%)$ a niños que consultaron poco y con los que no fue posible contactar por teléfono (por error en los datos de filiación). Otros se negaron a participar aduciendo falta de tiempo o de interés (5\%).

Tabla 1. Resultados del test de alimentación

\begin{tabular}{lc}
\hline & Porcentaje \\
\hline Utiliza aceite de oliva en casa & 95,3 \\
\hline Desayuna un lácteo (leche, yogur) & 93,1 \\
\hline Consume legumbres más de 1 vez por semana & 87,7 \\
Toma pasta o arroz 5 días o más por semana & 85,5 \\
\hline Toma pescado al menos 2 veces por semana & 81,1 \\
\hline Toma una fruta o zumo de fruta todos los días & 79,3 \\
\hline Desayuna un cereal o derivado & 77,8 \\
\hline Toma verduras frescas o cocinadas una vez al día & 66,5 \\
Toma 2 yogures y/o 40 gramos de queso al día & 53,5 \\
\hline Toma una segunda fruta todos los días & 35,5 \\
\hline Toma frutos secos al menos 2-3 veces por semana & 26,2 \\
\hline Desayuna bollería industrial & 16,0 \\
\hline Toma verduras más de una vez al día & 15,6 \\
\hline Toma golosinas varias veces al día & 14,5 \\
\hline No desayuna & 5,8 \\
\hline Acude 1 vez o más por semana a un restaurante de comida rápida & 4,7 \\
\hline
\end{tabular}


Tabla 2. Resultado global del test de alimentación

\begin{tabular}{lc} 
& Porcentaje \\
\hline Dieta mediterránea óptima & 52,3 \\
\hline Dieta de calidad media & 43,3 \\
\hline Dieta de muy baja calidad & 4,4 \\
\hline
\end{tabular}

La distribución por sexos fue homogénea (50\% para cada grupo). La prevalencia del sobrepeso en ambos sexos fue del 11,59\% (IC 95\%: 7,81\%-15,32\%) y la de la obesidad del 10,14\% (IC 95\%: 6,58\%-13,7\%).

En las tablas 1 y 2 se muestran los resultados obtenidos a partir del test sobre hábitos alimentarios, que revelan que el $52,3 \%$ de los niños sigue una alimentación mediterránea óptima y sólo un 4,4\% presenta hábitos totalmente inadecuados. También hay que destacar el alto consumo de legumbres $(88,7 \%)$, pasta y arroz (85,5\%), y la utilización mayoritaria del aceite de oliva (95,3\%).
Por otro lado, son bajos los porcentajes de quienes consumen una segunda fruta $(35,5 \%)$ o ración de verduras $(15,6 \%)$ a diario. No son irrelevantes los porcentajes de niños que no desayunan (5,8\%) o que lo hacen con bollería industrial (16\%).

En la tabla 3 se muestran los resultados referidos a la actividad física, que evidencian que una proporción importante $(35,9 \%)$ de la población estudiada no practica ninguna actividad deportiva extraescolar, y que un 51,8\% practica menos de 2 horas a la semana. Por otra parte, hasta un $63,4 \%$ invierte 2 o más horas al día en actividades sedentarias

\begin{tabular}{lccc}
\hline \multicolumn{4}{l}{ Tabla 3. Distribución porcentual de la población según el test de actividad física } \\
\hline Horas de deporte & Porcentaje & Horas de televisión & Porcentaje \\
\hline 0 & 35,9 & 0 & 2,2 \\
\hline 1 & 15,9 & 1 & 34,4 \\
\hline 2 & 21,4 & 2 & 41,7 \\
\hline 3 & 11,9 & 3 & 15,9 \\
4 & 9,1 & 4 & 4,4 \\
\hline 5 o más & 5,8 & 5 o más & 1,4 \\
\hline Total & 100 & Total & 100 \\
\hline
\end{tabular}

Resultado global del test de actividad física: mal estilo de vida: 58,7\%; estilo de vida regular: 37,7\%; buen estilo de vida: $3,6 \%$. 
como ver la televisión o jugar con videojuegos. De acuerdo con estos datos, debe considerarse que un $58,7 \%$ de la población presenta un mal estilo de vida y que sólo un $3,6 \%$ tiene uno bueno.

Respecto a las variables secundarias, cabe subrayar que un $10,9 \%$ de los niños de la muestra y un $15,6 \%$ de los padres han nacido en el extranjero, que el $22,5 \%$ de los progenitores no son convivientes y que un $33 \%$ de los niños son hijos únicos. El $58,7 \%$ de los padres tiene un nivel de instrucción bajo (sólo primaria o menos), mientras que en las madres el porcentaje es superior (63,3\%); esta diferencia es significativa ( $p=0,035)$. Existen también diferencias significativas $(p<0,001)$ entre el nivel de paro en los padres $(1,4 \%$ ) y las madres $(4,7 \%)$.

La información referida a la presencia de obesidad y/o sobrepeso en los progenitores muestra que un $41 \%$ de los padres presenta sobrepeso y un $12 \%$ obesidad, mientras que entre las madres los porcentajes son inferiores: un $23,4 \%$ tiene sobrepeso y un $10,4 \%$ obesidad; estas diferencias resultan significativas ( $p=$ $0,001)$.

De los niños de la muestra, el 72,5\% recibió lactancia materna con una duración media de 6,8 meses (IC 95\%: 5,85$7,74)$. La lactancia materna fue superior a 3 meses en el $64 \%$ de los casos y en el $10 \%$ superó los 13 meses.

El análisis bivariado no evidenció una relación estadísticamente significativa entre el IMC categorizado (existencia o no de sobrepeso/obesidad) y la mayoría de las variables estudiadas: actividad física (horas de deporte y de televisión), hábitos alimentarios, sexo, peso al nacer, lactancia materna, nivel de estudios de la madre y lugar de nacimiento de los niños o de los padres (tabla 4). Por el contrario, sí se han encontrado diferencias entre las medias del IMC de los niños cuando se han analizado las horas de televisión, el nivel de estudios de la madre

Tabla 4. Relación entre el índice de masa corporal categorizado y otras variables

\begin{tabular}{llllc}
\hline & & Normal & Sobrepeso/obesidad & p \\
\hline Horas de televisión & 2 o menos & $175(80,6 \%)$ & $42(19,4 \%)$ & NS \\
o videojuegos & 3 o más & $42(71,2 \%)$ & $17(28,8 \%)$ & \\
Estudios de la madre & Primaria o inferior & $129(75,4 \%)$ & $42(24,6 \%)$ & NS \\
& Secundaria o superior & $81(81,8 \%)$ & $18(18,2 \%)$ & \\
Lugar de nacimiento & Extranjero & $20(66,7 \%)$ & $10(33,3 \%)$ & NS \\
del niño & España & $197(80,1 \%)$ & $49(19,9 \%)$ & \\
\hline NS: no significativo. & & & & \\
\hline
\end{tabular}


Tabla 5. Relación entre el valor medio del índice de masa corporal y otras variables

\begin{tabular}{|c|c|c|c|c|}
\hline & & Media & $\mathrm{DE}$ & $p$ \\
\hline \multirow[t]{2}{*}{ Horas de televisión o videojuegos } & 2 o menos & 17,53 & 2,76 & 0,038 \\
\hline & 3 o más & 18,40 & 3,09 & \\
\hline \multirow[t]{2}{*}{ Estudios de la madre } & Primaria o inferior & 17,97 & 3,12 & 0,041 \\
\hline & Secundaria o superior & 17,28 & 2,35 & \\
\hline \multirow[t]{2}{*}{ Lugar de nacimiento del niño } & Extranjero & 18,74 & 2,88 & 0,036 \\
\hline & España & 17,59 & 2,83 & \\
\hline
\end{tabular}

DE: desviación estándar.

y el lugar de nacimiento del niño (tabla 5). En este sentido, las medias del IMC son mayores entre los que permanecen más inactivos, el nivel de estudios de la madre es bajo o nacieron fuera de España. La variable que más significativamente se encuentra relacionada con el IMC de los niños es la presencia de obesidad en los padres. Esta relación es patente cuando se realiza un análisis del IMC por categorías o como variable cuantitativa continua (tabla 6). Además, el IMC del niño sigue una correlación positiva del $29 \%$ y $26 \%$ con los IMC del padre y de la madre respectivamente, y estadísticamente significativa.

Según el modelo de regresión logística ajustado por sexo, edad y obesidad del padre o de la madre, el sobrepeso o la obesidad era 3,6 veces superior (IC 95\%: $1,37-8,05)$ si el padre era obeso, y 3,1 veces superior (IC 95\%: 1,19-7,31) si lo era la madre. También se observó que la edad de los niños se asociaba de forma positiva con la obesidad con una odds ratio de 1,4 (IC 95\%: 1,02-1,78).

\section{Discusión}

La muestra se calculó con una previsión de no respuestas del 10\%. Finalmente, este porcentaje ha sido superior, pero consideramos que el $81,4 \%$ de respuestas es aceptable para la finalidad de este estudio y que las características de las no respuestas no se relacionan con el objeto del estudio, excepto en un solo

Tabla 6. Relación entre el índice de masa corporal del niño y el de los padres

\begin{tabular}{lcccc}
\hline & $\begin{array}{c}\text { Normopeso } \\
\text { los dos }\end{array}$ & $\begin{array}{c}\text { Sobrepeso } \\
\text { los dos o uno }\end{array}$ & $\begin{array}{c}\text { Obesidad } \\
\text { los dos o uno }\end{array}$ & $p$ \\
Normopeso & $66(88 \%)$ & $122(78,2 \%)$ & $17(56,8 \%)$ & 0,002 \\
Sobrepeso + obesidad & $9(12 \%)$ & $34(21,8 \%)$ & $13(43,2 \%)$ & \\
\hline
\end{tabular}


caso conocido de obesidad y expreso rechazo de participar.

Los resultados de obesidad y sobrepeso (un $21,6 \%$ en su conjunto) obtenidos en el presente estudio son comparables a los de otros estudios realizados en ámbitos cercanos al nuestro. No obstante, constatamos en algunos casos que nuestras cifras de sobrepeso y obesidad son moderadamente inferiores a las observadas en otros estudios ${ }^{11-15}$. Esta diferencia puede tener diversas explicaciones: los grupos etarios no son exactamente los mismos en los diferentes estudios, las tablas utilizadas también pueden ser distintas y es posible que realmente la prevalencia sea diferente en nuestra zona. Hemos de considerar que, de un modo mayoritario, los niños de nuestra población han seguido el programa de salud infantil ${ }^{16}$ que desde principios de los años noventa se puso en marcha en nuestro centro. Por lo tanto, a la mayoría de los niños y familias del estudio ya se les ha ido proporcionando el consejo dietético que puede haber mejorado sus hábitos.

En la actualidad se plantea el carácter protector que la dieta mediterránea ejercería con relación al desarrollo de la obesidad, y algunos estudios, aunque no todos, así lo confirmarían ${ }^{17-20}$. En este sentido, podemos considerar que nuestra población presenta una aceptable adecuación a la mencionada dieta. En lo que respecta a la actividad física, varios estudios muestran que el sedentarismo y la disminución del gasto energético son factores decisivos en el desarrollo y la progresión de la obesidad ${ }^{8,21-23}$. En nuestro estudio, esta relación entre el ejercicio físico y la obesidad no ha podido constatarse, aunque sí se muestra que la media del IMC es mayor entre los niños que permanecen más tiempo realizando actividades sedentarias (televisión o videojuegos). Respecto al estilo de vida, que se refiere tanto al ejercicio como al sedentarismo, nuestra población presenta unos resultados sensiblemente mejorables, ya que sólo un 3,6\% presenta un estilo de vida óptimo y un $96,4 \%$ uno regular o malo. Si comparamos estos resultados con los obtenidos en el análisis de la actividad física del estudio enKid ${ }^{24}$ para la misma franja etaria $(23,1 \%$ con un estilo de vida óptimo y $76,9 \%$ con uno regular o malo), constatamos diferencias sustanciales que aconsejarían la puesta en marcha de programas para estimular la actividad física de los jóvenes y niños en detrimento del sedentarismo.

Además de los hábitos alimentarios y la actividad física, existen otros factores de riesgo que se han relacionado con la aparición de la obesidad en el 
niño $0^{25}$. Un factor de gran potencia predictiva es la obesidad o el sobrepeso de los padres ${ }^{26-28}$. También la mayoría de estudios, aunque no todos, establecen la lactancia materna y su duración como un factor protector de la obesidad infanti ${ }^{29-32}$. Otras variables que se mencionan son alto peso al nacer ${ }^{27,33}$, nivel socioeconómico y de instrucción bajos $^{34,35}$, crecimiento rápido en determinadas épocas de la infancia ${ }^{36,37}$, tabaquismo materno durante la gestación ${ }^{30,38}$ y horas de sueño (relación inversa) $^{33,39}$.

En el análisis bivariado efectuado que relaciona las variables principales y secundarias con el IMC se ha encontrado una correlación significativa entre la obesidad de los padres y la obesidad-sobrepeso de los niños. Como hemos señalado, esta relación ya ha sido advertida en múltiples estudios y ha de ayudarnos a la detección de niños en riesgo de obesidad. La falta de relación entre la mayoría del resto de variables y el IMC cabe atribuirla al propio diseño del estudio, pensado para establecer una línea de base descriptiva de nuestra población y, por tanto, sin efectivos poblacionales ni po- tencia estadística suficiente para esta finalidad.

\section{Conclusiones}

El presente estudio, además de establecer una valoración inicial de nuestra población, nos ha indicado algunos aspectos que se han tenido en cuenta al establecer las directrices y las actividades del programa de intervención comunitaria para la prevención de la obesidad infantil, puesto ya en marcha en nuestro centro. Estos aspectos son:

- Incidir en los hábitos alimentarios que consideramos mejorables: promover el consumo de frutas y verduras y la realización de un desayuno adecuado.

- Insistir en la promoción de la actividad física: reducir las actividades sedentarias, como ver la televisión o jugar con videojuegos, y estimular la actividad física en todas sus vertientes: escolar, extraescolar, deportiva y lúdica.

- Poner especial atención en aquellos niños que presenten factores de riesgo de obesidad, especialmente aquellos cuyos padres ya son obesos. 


\section{Bibliografía}

1. Foz G, Gofin J, Montaner I. Atención Primaria orientada a la Comunidad (APOC): una visión actual. En: Martín Zurro A, Cano Pérez JF, editores. Atención Primaria. Conceptos, organización y práctica clínica. 6. ${ }^{a}$ ed. Barcelona: Elsevier; 2008.

2. Carrascosa A. Obesidad durante la infancia y adolescencia. Una pandemia que demanda nuestra atención. Med Clin (Barc). 2006;126: 693-4.

3. Janssen I, Katzmarzy PT, Srinivasan SR, Chen W, Malina RM, Bouchard JC, et al. Comparison of overweight and obesity prevalence in school-ages youth from 34 countries and their relationships with physical activity and dietary patterns. Obes Rev. 2005;6:123-32.

4. Freedman DS, Dietz WH, Srinivasa SR, Berenson GS. The relation of overweight to cardiovascular risk factors among children and adolescents. The Bogalusa heart study. Pediatrics. 1999;103: 1175-82.

5. Must A, Jacques PF, Dallal GE, Bajema CJ, Dietz $\mathrm{WH}$. Long term morbidity and mortality of overweight adolescents. A follow-up of the Harvard Growth Study of 1922 to 1935. N Engl J Med. 1992;327:1350-5.

6. Hannon TS, Goutham R, Arslanian MD. Childhood obesity and Type 2 Diabetes Mellitus. Pediatrics. 2005;116:473-80.

7. Agencia Española de Seguridad Alimentaria. Ministerio de Sanidad y Consumo. Estrategia Naos para la nutrición, actividad física y prevención de la obesidad. Madrid: Ministerio de Sanidad y Consumo; 2005.

8. Aranceta Bartrina J, Pérez Rodrigo C, Ribas Barba L, Serra Majem L. Epidemiología y factores determinantes de la obesidad infantil y juvenil en España. Rev Pediatr Aten Primaria. 2005;7 (supl 1):s13-s20.
9. Dossier de Consenso. Curvas de referencia para la tipificación ponderal. Población infantil y juvenil. AEP, SENC, SEEDO. Madrid: IMC; 2002. p. 71-80.

10. Serra Majem L, Aranceta Bartronaq J, Ribas Barba L, Sangil Monroy M, Pérez Rodrigo C. El cribado del riesgo nutricional en pediatría. Validación del test rápido Krece Plus y resultados en la población española. En: Estudio enKid. Crecimiento y desarrollo. Barcelona: Masson; 2003. p. 45-55.

11. Martínez Vizcaino V, Salcedo Aguilar F, Franquelo Gutiérrez R, Torrijos Regidor R, Morant Sánchez A, Solera Martínez M, y cols. Prevalencia de obesidad y tendencia de los factores de riesgo cardiovascular en escolares de 1992 a 2004: estudio de Cuenca. Med Clin (Barc). 2006; 126:681-5

12. Serra Majem L, Aranceta Bartrina J, Pérez Rodrigo C, Ribas Barba L, Delgado Rubio A. Prevalence and determinants of obesity in Spanish children and young people. Br J Nutr. 2006;96 (suppl 1):s67-s72.

13. Moreno LA, Mesana MI, Fleta J, Ruiz JR, González-Gross $M$, Sarria $A$, et al. Overweight, obesity and body fat composition in Spanish adolescents. The AVENA Study. Ann Nutr Metab. 2005;49:71-6.

14. Neira $M$, De Onis $M$. The Spanish strategy for nutrition, physical activity and the prevention of obesity. Br J Nutr. 2006;96 (Suppl 1):s8-s11.

15. Albañil Ballesteros $M R$, Sánchez Martín $M$, De la Torre Verdú $M$, Olivas Domínguez A, Sánchez Méndez MY, Sanz Cuesta T. Prevalencia de obesidad a los 14 años en cuatro consultas de atención primaria. Evolución desde los dos años. An Pediatr (Barc). 2005;63:39-44.

16. Protocols de Medicina Preventiva en l'edat pediàtrica. Programa de Seguiment del Nen Sa. Barcelona: Departament de Sanitat i de Seguretat Social, Generalitat de Catalunya; 1995. 
17. Panagiotakos DB, Chrysohoou C, Pitsavos C, Stefanadis C. Association between the prevalence of obesity and adherence to the Mediterranean diet: the ATTICA Study. Nutrition. 2006;22: 449-56.

18. Schröder H, Marrugat J, Vila J, Covas MI, Elosua R. Adherence to the traditional Mediterranean diet is inversely associated with body mass index and obesity in a Spanish population. J Nutr. 2004;134:3355-61.

19. Hassapidou M, Fotiadou E, Maglara E, Papadopoulou SK. Energy intake, diet composition, energy expeditura, and body fatness of adolescents in northern Greece. Obesity. 2006;14:855-62.

20. Auckland G, Bach A, Serra Majem L. Obesity and the Mediterranean diet: a systematic review of observational and intervention studies. Obes Rev. 2008:9:582-93.

21. Bautista Castaño I, Sangil Monroy M, Serra Majem L. Conocimientos y lagunas sobre la implicación de la nutrición y la actividad física en el desarrollo de la obesidad infantil. Med Clin (Barc). 2004;123:782-93.

22. Giugliano R, Carneiro EC. Factors associates with obesity in school children. J Pediatr (Rio J). 2004;89:1-2.

23. Lumeng JC, Rahnama S, Appugliese D, Kaciroti N, Bradley RH. Television exposure and overweight risk in preschoolers. Arch Pediatr Adolesc Med. 2006;160:417-22.

24. Román Viñas B, Serra Majem L, Ribas Barba L, Pérez Rodrigo C, Aranceta Bartrina J. Estimación del nivel de actividad física mediante el Test Corto Krece Plus. Resultados de la población española. En: Crecimiento y desarrollo. Estudio enKid. Barcelona: Masson; 2003. p. 59-74.

25. Krebs NF, Jacobson MS, American Academy of Pediatrics Committee on Nutrition. Prevention of pediatric overweight and obesity. Pediatrics. 2003;112:424-30.
26. Mamun AA, Lawlor DA, O'Callaghan MJ, Williams GM, Najman JM. Family and early life factors associated with changes in overweight status between ages 5 and 14 years: findings from the Mater University Study of Pregnancy and its outcomes. Int J Obes (Lond). 2005;29:475-82.

27. Hui LL, Nelson EA, Yu LM, Li AM, Fok TF. Risk Factors for childhood overweight in 6-to 7-yold Hong Kong children. Int J Obes Relat Metab Disord. 2003;27:1411-8.

28. Danielzik $S$, Langnäse $K$, Mast $M$, Spethmann $C$, Müller MJ. Impact of parental BMI on the manifestation of overweight 5-7 year old children. Eur J Nutr. 2002;41:132-8.

29. Grummer-Strawn LM, Mei Z. Does breastfeeding protect against pediatric overweight? Analysis of longitudinal data from the centers for disease control and prevention pediatric nutrition surveillance system. Pediatrics. 2004;113: 81-6.

30. Bergmann KE, Bergmann RL, Von Kries R, Böhm O, Richter R, Dudenhausen JW, et al. Early determinants of childhood overweight and adiposity in a birth cohort study: role of breast-feeding. Int J Obes. 2003;27:162-72.

31. Harder $T$, Bergmann $R$, Kallischnigg $G$, Plagemann $A$. Duration of breastfeeding and risk of overweight: a meta-analysis. Am J Epidemiol. 2005;162:397-403.

32. Owen CG, Martin RM, Whincup PH, Smith $G D$, Cook DG. Effect of infant feeding on the risk of obesity across the life course. A quantitative review of published evidence. Pediatrics. 2005;115: 1367-77.

33. Padez C, Mouräo P, Rosado V. Prevalence and risk factors for overweight and obesity in Portuguese children. Acta Pediatr. 2005;94:1550-7.

34. Veugelers PJ, Fitzgerald AL. Prevalence of and risk factors for childhood overweight and obesity. CMAJ. 2005;173:607-13. 
35. Lamerz A, Kuepper-Nybelen J, Wehle C, Bruning N, Trost-Brinkhues $\mathrm{G}$, Brenner $\mathrm{H}$, et al. Social class, parental education and obesity prevalence in a study of six-year-old children in Germany. Int J Obes (Lond). 2005;29:373-80.

36. Baird J, Fisher D, Lucas P, Kleijnen J, Roberts $H$, Law C. Being big or growing fast: systematic review of size and growth in infancy and later obesity. BMJ. 2005;331:929.

37. Reilly J, Armtrong J, Dorosty A, Emmett PM, Ness A, Rogers I. Early life risk factors for obesity in childhood: cohort study. BMJ. 2005; 330:1357.

38. Salsberry PJ, Reagan PB. Dynamics of early childhood overweight. Pediatrics. 2005;116:132938.

39. Lumeng JC, Somashekar D, Appugliese D, Kaciroti N, Corwyn RF, Bradley RH. Shorter sleep duration is associated with increased risk for being overweight at 9 to 12 years. Pediatrics. 2007;120: 1020-9. 